\title{
Melting Behavior of Bound Solvent in Isotactic Polypropylene/o-Dichlorobenzene Gel
}

\author{
Takahiko NAKAOKI ${ }^{\dagger}$ and Shingo HARADA \\ Faculty of Science and Technology, Department of Materials Chemistry, Ryukoku University, \\ Seta, Otsu 520-2194, Japan
}

(Received January 11, 2005; Accepted February 22, 2005; Published June 15, 2005)

\begin{abstract}
The melting behavior of $o$-dichlorobenzene in isotactic polypropylene (iPP)/o-dichlorobenzene gel was investigated by differential scanning calorimetry (DSC) and polarizing microscopy. A DSC thermogram provided two endothermic peaks for the melting of $o$-dichlorobenzene in the gel. The high melting temperature was almost the same as for pure $o$-dichlorobenzene. Therefore the solvent can be regarded as being like free solvent. However it shifted slightly to higher temperature depending on polymer concentration, indicating that the solvent was weakly bound in the gel. The low melting peak was observed at a few tens degree lower than the high melting peak. The low melting peak was very broad and decreased depending on concentration from $-17.7^{\circ} \mathrm{C}$ for the $5 \mathrm{wt} \%$ gel to $-34.1^{\circ} \mathrm{C}$ for the $30 \mathrm{wt} \%$ gel. The solvent in the gel concentrated from 10 to $60 \mathrm{wt} \%$ through solvent evaporation provided only one broad peak corresponding to the low melting peak around $-39^{\circ} \mathrm{C}$. That no high melting peak was observed shows that the solvent with high melting temperature was weakly bound in the gel and was excluded with ease from the gel. Therefore the low melting peak corresponds to the melting of more strongly bound solvent. This indicates two bound solvent molecules in the gel, one loosely bound in the gel and the other bound more firmly by the polymer. In the $2 \mathrm{wt} \%$ gel, the polarinzing microscopy showed that the spherulites were isolated from others. This suggests that the solvent with high melting peak locates in the space between spherulites. The solvent in this region would be close to free solvent. The spherulites for the concentrated gel contacted adjacent ones. In this case, the molecular chains are so distorted between spherulites that the solvent molecule is weakly bound in this boundary region. This provides the high melting peak slightly shifted to higher temperature. The low melting temperature was significantly lowered depending on polymer concentration. The solvent would be strongly bound in noncrystalline phase or interphase between lamellar crystals in the spherulite. [DOI 10.1295/polymj.37.429]

KEY WORDS Isotactic Polypropylene / Gel / DSC / Bound Solvent / Morphology /
\end{abstract}

Thermal analysis is useful for investigating not only melting behavior and phase transition of bulk polymer but also sol-gel transition. Gelation of a crystalline polymer such as isotactic polypropylene (iPP) is closely associated with the formation of crystal as a cross-linking point. ${ }^{1-5}$ Okabe et al. reported a solgel transition and the morphology of iPP gel by thermal analysis and polarizing microscopy, ${ }^{6,7}$ and found that the gel melting temperature is fitted as a function of polymer concentration by a Eldridge-Ferry plot and many spherulites exist in the gel. There exist three types of crystalline modification. ${ }^{8-11}$ The $\alpha$-form with monoclinic cell is the most stable crystalline form. Studies on the crystalline structure formed in iPP/ $o$-dichlorobenzene gel have been reported by high resolution solid state ${ }^{13} \mathrm{C}$ NMR measurements. ${ }^{3,4} \mathrm{~A}$ cross polarization $(\mathrm{CP}) /$ magic angle spinning (MAS) spectrum showed the characteristic doublets for methylene and methyl resonance lines with an intensity ratio of 1:2 from downfield, whereas the stable crystalline $\alpha$-form ${ }^{12-18}$ provided a spectrum with the opposite intensity ratio. The gel-forming crystal possibly takes a disordered form. ${ }^{4}$
Most investigations on gel have focused on details of the sol-gel transition, but some are concerned with the coagulation and melting behavior of the solvent in the gel. Higuchi et al. investigated the melting behavior of water in a polyvinyl alcohol (PVA) swollen gel, and showed the existence of free water and freezable bound water. ${ }^{19}$ Nishinari et al. carried out thermal analysis of the water in concentrated agarose gel. ${ }^{20}$ The melting of water was characterized by two endothermic peaks. They suggested that the lower melting temperature peak corresponds to the melting of disordered frozen water and the higher one to the melting of more stable water in the form of hexagonal ice crystals. Yoshida et al. showed the temperature dependence of the X-ray diffraction pattern for a hyaluronic acid hydrogel and concluded that the crystal melting just below the $T_{\mathrm{m}}$ of stable water consists of monoclinic ice crystals. ${ }^{21}$ These investigations were all concerned with hydrogels.

This paper investigates the melting behavior of the organic solvent in iPP gel. The absence of hydrogen bonding in these systems is noteworthy and might be expected to result in unusual melting behavior of

${ }^{\dagger}$ To whom correspondence should be addressed (E-mail: nakaoki@rins.ryukoku.ac.jp). 
free and bound solvent molecules in the organic gel.

\section{EXPERIMENTAL}

A sample of iPP was purchased from Showa Denko Co. Ltd. and purified by soxlet extraction with $n$-heptane. The weight average molecular weight $\left(M_{\mathrm{w}}\right)$ and meso triad (mm) were estimated to be 212,000 and $98.5 \%$, respectively. Gel was prepared as follows. iPP and $o$-dichlorobenzene were placed in an ampoule, degassed in vacuo and sealed. The solution was homogeneously dissolved at $170^{\circ} \mathrm{C}$, and then quenched in ice-water. Gels were kept in ice-water for $24 \mathrm{~h}$ to produce a stable gel. Partially dried gel was prepared by evaporating the solvent to obtain desired weight concentration at room temperature for a few weeks.

Thermal analysis was performed using a Rigaku TAS 200 differential scanning calorimeter (DSC). The samples were scanned at a heating rate of $5^{\circ} \mathrm{C} /$ min under a nitrogen flow. Calibration of the instrument for temperature was accomplished with $\mathrm{CHCl}_{3}$. An aluminum sealed pan was used for gel samples. Measurements were carried out at least three times between $-100^{\circ} \mathrm{C}$ and $10^{\circ} \mathrm{C}$ and the average maximum temperature of the peaks was adopted. Polarizing microscopic measurement was carried out at room temperature by Olympus BX51TF. The diameter of spherulite was averaged for more than ten spherulites.

\section{RESULTS AND DISCUSSION}

Water in a hydrogel takes free or bound state. The different environments for the water molecule in the coagulated polymer chain result in different melting temperatures. In contrast to a hydrogel, the absence of hydrogen bonding in an organogel may result in different melting behavior of the organic solvent. To investigate the possible occurrence of a different melting behavior of $o$-dichlorobenzene in iPP/o-dichlorobenzene gel, we measured DSC thermogram in the temperature range from $-100^{\circ} \mathrm{C}$ to $10^{\circ} \mathrm{C}$. Figure 1 shows the DSC chart of a $20 \mathrm{wt} \%$ gel along with the pure $o$-dichlorobenzene. For the gel, a broad endothermic peak was observed at $-26.5^{\circ} \mathrm{C}$ in addition to the main peak at $-12.5^{\circ} \mathrm{C}$. The observation of two melting peaks for solvent provides a result similar for the hydrogel. The main peak was observed near the $T_{\mathrm{m}}$ of pure $o$-dichlorobenzene, and thus probably corresponds to the melting of free solvent or solvent close to a free state. To obtain further information on these melting peaks, we observed the melting behavior depending on polymer concentration. Figure 2 shows the DSC thermogram of iPP/o-dichlorobenzene gel of variable concentration. Two endothermic peaks of high and low melting temperatures were ob-

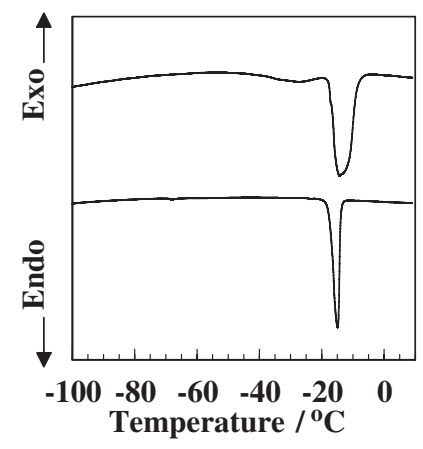

Figure 1. DSC thermogram of $20 \mathrm{wt} \%$ iPP/o-dichlorobenzene gel and pure $o$-dichlorobenzene.

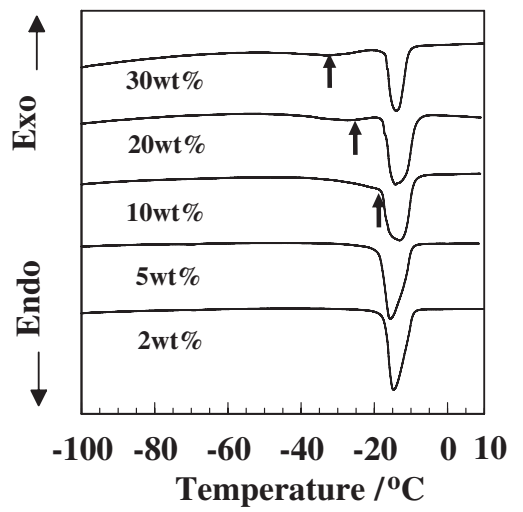

Figure 2. Concentration dependence of DSC thermograms for iPP/o-dichlorobenzene gels. Low melting peaks are indicated by arrows.

served for all gels; the former was sharp and the latter was very broad. The high melting peak of the $2 \mathrm{wt} \%$ gel was observed at $-15.4^{\circ} \mathrm{C}$, which is close to the $T_{\mathrm{m}}$ of pure $o$-dichlorobenzene. With increasing polymer concentration, the high melting peak was broadened accompanied by a higher temperature shoulder. The peak maximum temperature is plotted as closed circles in Figure 3. The peak maximum shifted slightly to higher temperature with increasing polymer concentration. This suggests that the solvent exists in close to a free state in the low concentration gel, but with increasing concentration the solvent tends to take the bound state. However, the solvent is not strongly bound, because the maximum temperature was always observed near the $T_{\mathrm{m}}$ of pure solvent.

The temperature of the low melting peak, shown as filled squares in Figure 3, shifted considerably toward low temperature with increasing polymer concentration. The peak temperature for the $30 \mathrm{wt} \%$ gel is $-34.1^{\circ} \mathrm{C}$, whereas that of the $5 \mathrm{wt} \%$ gel is $-17.7^{\circ} \mathrm{C}$, a temperature difference of almost $17^{\circ} \mathrm{C}$. One reason for this strong dependence on polymer concentration is that the solvent may be in a non-equilibrium state due to interactions such as residual strain. To confirm 


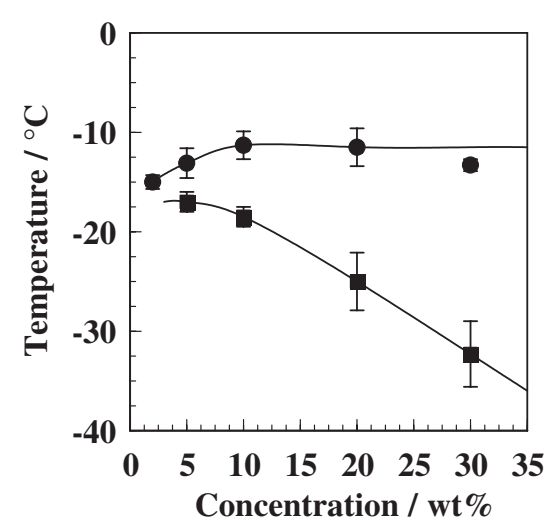

Figure 3. Melting temperature of $o$-dichlorobenzene in the gel as a function of polymer concentration. ๑: high melting temperature, $\mathbf{\square}$ : low melting temperature.

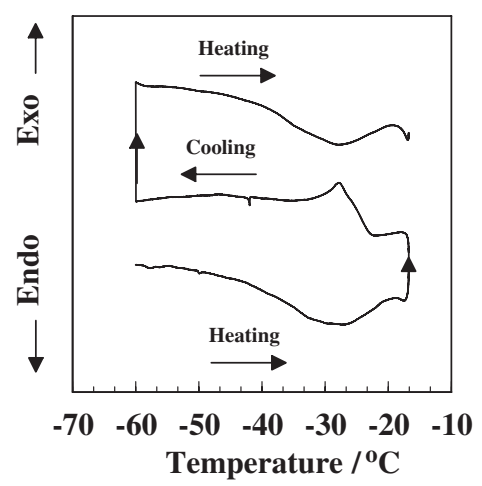

Figure 4. DSC thermogram of $20 \mathrm{wt} \% \mathrm{iPP} / o$-dichlorobenzene gel. The first heating process was stopped at $-17^{\circ} \mathrm{C}$. After cooling to $-60^{\circ} \mathrm{C}$, the second heating process was started.

the thermal reversibility of this peak, a cyclic DSC measurement was carried out for the $20 \mathrm{wt} \%$ gel, as shown in Figure 4. The first run was performed from $-60^{\circ} \mathrm{C}$ to $-17^{\circ} \mathrm{C}$. The broad endothermic peak was observed around $-26^{\circ} \mathrm{C}$. After cooling to $-60^{\circ} \mathrm{C}$, heating was repeated, and a low melting peak was clearly reproduced. This indicates that the low melting peak is thermally reversible. The melting temperature considerably shifts depending on the polymer concentration, it may indicates close relation between the bound state of solvent and the morphology of polymer chain.

\section{Melting Behavior of the Gel Concentrated through Drying Process}

To confirm how strongly the solvent is bound in the gel, the $10 \mathrm{wt} \%$ gel was allowed to stand for a few weeks, and the solvent was evaporated in the draft chamber. The gel was concentrated until the polymer concentration became 30,60, and $90 \mathrm{wt} \%$ and DSC measurements were carried out. Heating between $-100^{\circ} \mathrm{C}$ and $10^{\circ} \mathrm{C}$ is shown in Figure 5. For the gel

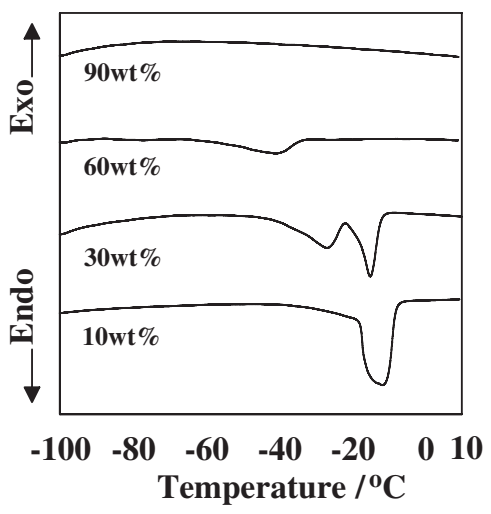

Figure 5. DSC thermogram of 30,60 , and $90 \mathrm{wt} \%$ gels evapolated from $10 \mathrm{wt} \%$ gel.

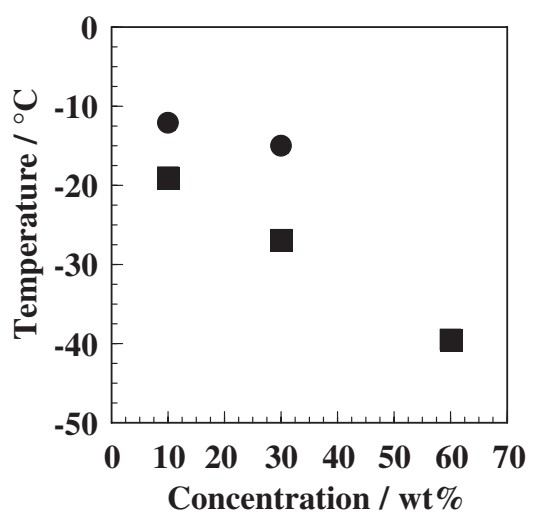

Figure 6. Melting temperature of high and low endothermic peaks depending on concentration after evaporation.

concentrated to $30 \mathrm{wt} \%$, two endothermic peaks were observed. The high melting peak was close to that for $10 \mathrm{wt} \%$ gel, but it became small in intensity. The low melting peak shifted toward low temperature compared with that for the $10 \mathrm{wt} \%$ gel. In the DSC chart of the gel concentrated to $60 \mathrm{wt} \%$, there was no trace of high melting peak, but only the low melting peak was observed at $-39^{\circ} \mathrm{C}$. The gel concentrated to 90 wt $\%$ gave no peaks. The solvent with high melting temperature was thus evaporated with ease during drying process, whereas that with low melting temperature took a long time to evaporate. The solvents with high and low melting temperatures are thus weakly and strongly bound in the gel. In Figure 6 plotted the endothermic temperature as a function of polymer concentration after evaporating the solvent. The low melting peak shifted toward low temperature with polymer concentration. The temperature shift of concentrated gel can be identified with the low melting temperature shown in Figure 2.

A possible explanation for the formation of two bound solvents would be related with the morphology 

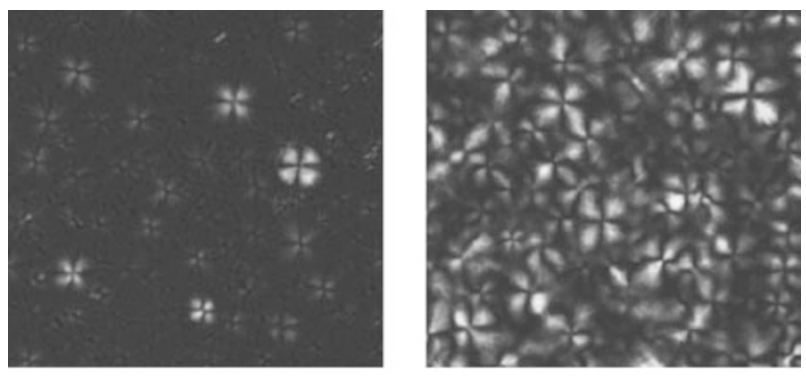

2 wt $\%$

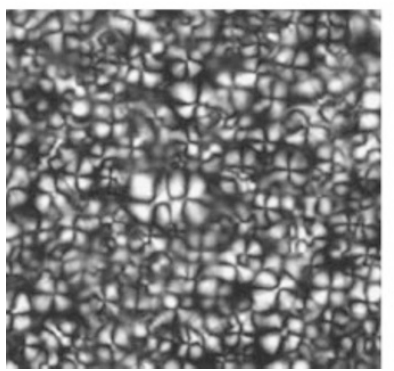

$10 \mathrm{wt} \%$

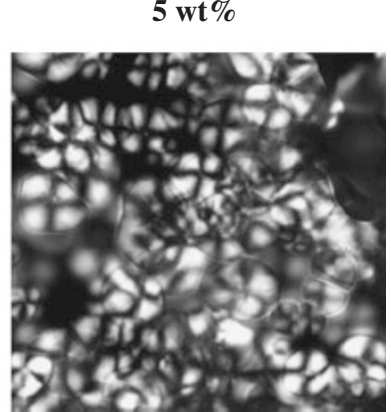

$20 \mathrm{wt} \%$

Figure 7. Polarizing microscopic photograph for various concentrations of the $\mathrm{iPP} / \mathrm{o}$-dichlorobenzene gel formed at $0{ }^{\circ} \mathrm{C}$.

of the polymer chain. Okabe et al. reported that the spherulite is formed in iPP gel. ${ }^{1,2}$ The weakly bound solvent might be bound in the distorted noncrystalline chains, whereas the strongly bound solvent might be bound in more strained phase adjacent to crystalline region. Therefore we discuss the polarizing microscopic photograph of the iPP gel as a function of polymer concentration in the next section.

\section{Polarizing Microscopic Photographs of Various Con- centration of Gel}

To clarify the relationship between molecular morphology and the solvent bound in the gel, polarizing microscopy was carried out. Figure 7 shows the microscopic photograph of the $2,5,10$, and $20 \mathrm{wt} \%$ gels. The maltese cross was clearly observed for all gels, indicating the formation of spherulites. With polymer concentration, the number of spherulite increased and the spherulite size became larger. Since the solvent cannot be put in the crystalline region, ${ }^{3}$ the space that the solvent can exist would be limited to noncrystalline region between the sperulites or in the spherulite. The spherulites in the $2 \mathrm{wt} \%$ gel were isolated from others as shown in Figure 4, corresponding to the very large space between spherulites. Since the polymer chain between spherulites is basically regarded as noncrystalline, the solvent in this region is weakly bound, although the solvent would be close to free state so that the high melting peak was broader than the melting peak of the pure solvent.

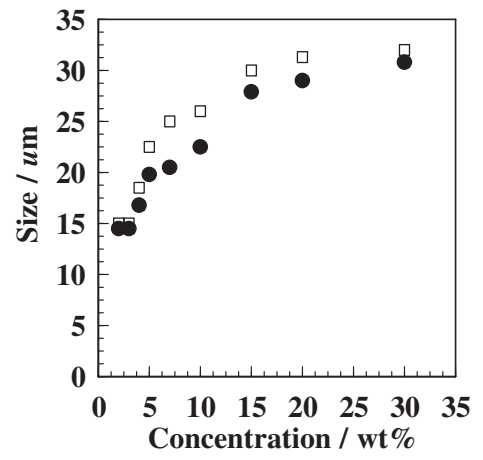

Figure 8. Diameter of a spherulite depending on polymer concentration.

The spherulites for higher concentration gel contacted adjacent ones. Therefore the polymer chain between spherulites would be distorted more than the low concentration gel. The solvent in this region takes essentially the same state as the gel formed from dilute solution such as $2 \mathrm{wt} \%$ but the bound state would be a little stronger. This corresponds that the high melting peak in DSC measurement tends to shift toward high temperature.

The low melting peak was assumed due to the melting of strongly bound solvent as shown in previous section. The solvent molecules would be located in the spherulite. In general, a spherulite consists of three phases of crystalline lamella, noncrystalline phase, and interphase between crystalline and noncrystalline. ${ }^{22,23}$ Although the solvent can be trapped in noncrystalline and interphase, most of the solvent would be in the interphase due to strongly bound state. The solvent molecules in this region would not easily form large crystalline domain so that the melting temperature is lower. In Figure 8, the average diameter of spherulites is plotted against polymer concentration. The diameter of $2 \mathrm{wt} \%$ gel was around $15 \mu \mathrm{m}$, whereas that of $30 \mathrm{wt} \%$ became $30 \mu \mathrm{m}$. Spherulite size may thus be related to temperature shift of the low melting peak in DSC measurement. Perhaps the solvent in large spherulite is strongly trapped in the distorted interphase between lamellar, and the melting temperature is lowered.

\section{CONCLUSIONS}

Thermal analysis and polarizing microscopic measurements were carried out to elucidate the melting behavior of $o$-dichlorobenzene in iPP/o-dichlorobenzene gel. This system is suitable for investigating a bound solvent because of the absence of hydrogen bonding. For the $20 \mathrm{wt} \%$ gel, two melting peaks at $-12.5^{\circ} \mathrm{C}$ and $-26.5^{\circ} \mathrm{C}$ were observed by DSC measurement. Since the former temperature is close $T_{\mathrm{m}}$ of 
pure $o$-dichlorobenzene, the solvent is free or weakly bound. The temperature of the low melting peak had strong dependence on concentration. The peak maximum shifted from $-34.1^{\circ} \mathrm{C}$ for a $30 \mathrm{wt} \%$ gel to $-17.7^{\circ} \mathrm{C}$ for a $5 \mathrm{wt} \%$ gel. This endothermic peak is regarded as a reversible transition, since this peak was reproducible by DSC measurement. The bound state in the gel was investigated by the gel formed through solvent evaporating process. After preparing the $10 \mathrm{wt} \%$ gel, the solvent was evaporated to be 30,60 , and $90 \mathrm{wt} \%$ gels. In the thermogram of the gel concentrated to $30 \mathrm{wt} \%$, the peak intensity of high melting peak became small. For the dried $60 \mathrm{wt} \%$ gel, this peak was not observed but there was only low melting peak. Thus the solvent with high melting temperature is weakly bound in the gel and evaporates with ease, whereas the solvent with low melting temperature is strongly bound in the gel and takes a long time to evaporate.

Polarinzing microscopic measurement was carried out to elucidate the relation between molecular morphology and bound state of the solvent. The maltese cross was observed for all gels, indicating the formation of spherulite. The spherulites in $2 \mathrm{wt} \%$ gel were isolated from others, leading to large spaces between spherulites. The solvent in this region would behave like free solvent. As a result, the melting temperature was almost identified with that of pure solvent. The solvent with low melting temperature would locate between lamellar in the spherulite due to the strongly bound solvent. For larger spherulites, the melting temperature became low. Since the spherulite was strongly packed by crystalline lamellar, the solvent would be trapped in the interphase between lamellar. These show that the morphology of polymer chain played an important role to determine the melting behavior of solvent.

Acknowledgment. This work was financially supported by the High Tech Research Center at Ryukoku University, Japan.

\section{REFERENCES}

1. H. Matsuda, T. Inoue, and M. Okabe, Rep. Prog. Polym. Phys. Jpn., 29, 33 (1986).

2. H. Matsuda, T. Inoue, M. Okabe, and T. Ukaji, Polym. J., 19, 323 (1987).

3. T. Nakaoki, H. Shuto, H. Hayashi, and R. Kitamaru, Polymer, 39, 3905 (1998).

4. T. Nakaoki and Y. Inaji, Polym. J., 34, 539 (2002).

5. M. Matsuo, T. Hashida, K. Tashiro, and Y. Agari, Macromolecules, 35, 3030 (2002).

6. G. Natta, P. Pino, P. Corradini, F. Danuss, E. Mantica, G. Mazzanii, and G. Moriglio, J. Am. Chem. Soc., 77, 1708 (1955).

7. G. Natta and P. Corradini, Nuovo Cimento Suppl., 15, 40 (1960).

8. S. V. Meille, D. R. Ferro, S. Bruckner, A. J. Lovinger, and F. J. Padden, Macromolecules, 27, 2615 (1994).

9. B. Lots, S. Kopp, and D. Dorset, C. R. Acad. Sci. Paris, Ser. Iib, 319, 187 (1994).

10. S. Bruckner and S. V. Meille, Nature, 340, 455 (1989).

11. S. V. Meille, S. Bruckner, and W. Porzio, Macromolecules, 23, 4114 (1990).

12. M. A. Gomez, H. Tanaka, and A. E. Tonelli, Polymer, 28, 2227 (1987).

13. V. Caldas, G. R. Brown, R. S. Nohr, J. G. MacDonald, and L. E. Raboin, Polymer, 35, 899 (1994).

14. V. Caldas, F. G. Morin, and G. R. Brown, Magn. Reson. Chem., 32, S72 (1994).

15. V. Caldas, G. R. Brown, R. S. Nohr, and J. G. MacDonald, J. Polym Sci., Part B: Polym. Phys., 34, 2085 (1996).

16. A. Bunn, M. E. A. Cudby, R. K. Harris, K. J. Packer, and B. J. Say, J. Chem. Soc., Chem. Commun., 15 (1981).

17. A. Bunn, M. E. A. Cudby, R. K. Harris, K. J. Packer, and B. J. Say, Polymer, 23, 694 (1982).

18. S. Saito, Y. Moteki, M. Nakagawa, F. Horii, and R. Kitamaru, Macromolecules, 23, 3256 (1990).

19. A. Higuchi and T. Iijima, Polymer, 26, 1207 (1985).

20. M. Watase, K. Nishinari, and T. Hatakeyama, Food Hydrocolloids, 2, 427 (1988).

21. H. Yoshida, T. Hatakeyama, and H. Hatakeyama, Polym. Prepr., Jpn., 41, 3032 (1992).

22. P. J. Flory, J. Chem. Phys., 17, 223 (1949).

23. P. J. Flory, J. Am. Chem. Soc., 84, 2587 (1962). 